\section{Dietary supplementation with vitamins, minerals, and fatty acids reduced antisocial behaviour in young adult prisoners}

\author{
Gesch CB, Hammond SM, Hampson SE, et al. Influence of supplementary vitamins, minerals and essential fatty acids on \\ the antisocial behaviour of young adult prisoners. Randomised, placebo-controlled trial. Br J Psychiatry 2002;181:22-8.
}

\section{QUESTION: In young adult prisoners, does supplementation with vitamins, minerals, and fatty acids reduce antisocial behaviour?}

\section{Design}

Randomised \{allocation concealed*\}†, blinded (clinicians, participants, outcome assessors, \{data collectors, data analysts, and data safety monitoring committee $\} \uparrow),{ }^{*}$ placebo controlled trial with mean 142 days of follow up.

\section{Setting}

\{HM Young Offenders Institution, a maximum security institution in Aylesbury, UK\}†.

\section{Participants}

231 prisoners who were $\geq 18$ years of age mean age 19 $\mathrm{y}, 100 \%$ men $\}$. Follow up was $100 \%$ for the intention to treat analysis.

\section{Intervention}

After stratification for prison wing, participants were allocated to dietary supplementation $(\mathrm{n}=116)$ or placebo $(\mathrm{n}=115)$. Dietary supplementation consisted of a vitamin and mineral supplement, Forceval, and a fatty acid supplement, Efamol Marine, given in 4 daily capsules (linoleic acid, $1260 \mathrm{mg}$; gamma linolenic acid, $80 \mathrm{mg}$; eicosapentaenoic acid, $80 \mathrm{mg}$; and docosahexaenoic acid, $44 \mathrm{mg}$ ). The supplements matched the UK government's recommended dietary intakes for vitamins, minerals, and essential fatty acids.

\section{Main outcome measure}

Antisocial behaviour resulting in disciplinary action (Governor reports of more serious incidents, such as violence, and minor reports [eg, failure to comply with requirements]).

\section{Main results}

Analysis was by intention to treat. 532 Governor reports and 601 minor reports were made during the study period. Participants who received supplements committed fewer infringements than those who received placebo (2 tailed $\mathrm{p}=0.03$ ) (table). No adverse events were reported.

\section{Conclusion}

In young adult prisoners, supplementation with vitamins, minerals, and fatty acids reduced antisocial behaviour.
*See glossary.

$\dagger$ Information provided by author.

Source of funding:

Natural Justice.

Nutritional

supplements supplied

by Scotia

Pharmaceuticals Ltd

and Unigreg Ltd.

For correspondence:

Mr C B Gesch,

University of Oxford,

Oxford, UK.

bernard.gesch@

physiol.ox.ac.uk

Dietary supplementation (vitamins, minerals, and fatty acids) v placebo in young adult prisoners

\begin{tabular}{|c|c|c|c|}
\hline \multirow{2}{*}{$\begin{array}{l}\text { Outcome at mean } 142 \\
\text { days }\end{array}$} & \multicolumn{2}{|c|}{$\begin{array}{l}\text { Mean number of infringements per } \\
1000 \text { person-days }\end{array}$} & \multirow{2}{*}{$\begin{array}{l}\text { Mean percentage } \\
\text { reduction }(95 \% \mathrm{Cl})\end{array}$} \\
\hline & Supplements & Placebo & \\
\hline $\begin{array}{l}\text { Antisocial behaviour } \\
\text { resulting in } \\
\text { disciplinary action }\end{array}$ & 11.8 & 16.0 & $26 \%(8.3$ to 44$)$ \\
\hline
\end{tabular}

$\ddagger$ Difference between groups favours dietary supplementation.

\section{COMMENTARY}

The idea that we are what we eat has a long currency in popular culture. Dietary treatments are frequently recommended across a range of clinical disorders, particularly those that are difficult to understand and treat. There seems little doubt that pathologically aggressive behaviour is linked to poor nutrition. ${ }^{1}$ However, important correlations with other risk factors such as social and economic deprivation, low educational attainment, and substance misuse ${ }^{2}$ mean that in this field the proof of the pudding really is in the eating. The study by Gesch et al has high marks on this score because it is prospective, randomised, double blind, and placebo controlled. Most obvious confounders, such as differences in baseline levels of offending and dietary intake together with placement in the institution, seem to have been identified and resolved. The use of nutritional supplements to treat aggression is also supported by another controlled trial in delinquent schoolchildren in the US. ${ }^{1}$

In both of these studies, a broad spectrum of minerals and vitamins was provided by the dietary treatment, and Gesch $e t$ al also added essential fatty acid supplementation. Hence we cannot be sure which, if any, of the ingredients played a critical role in the treatment effect or whether the therapeutic activity was conveyed by the complex combination of nutrients. The participants in the study by Gesch $e t$ al seemed to have nutritional intakes below UK reference nutrient intakes (RNIs). Therefore another important question is whether nutritional treatment of this nature will be effective only in those with inadequate diets. However, it needs to be remembered that RNI levels have been determined largely on the basis of the amounts of vitamins and minerals required to prevent overt clinical deficiency disorders. Hence, it is possible that even in those with apparently adequate dietary intakes, nutritional supplementation could produce subtle neuropsychological changes that could modify the expression of complex social behaviours such as pathological aggression. At any rate, the study by Gesch $e t$ al does support the use of nutritional supplementation in populations where inadequate dietary intake and behavioural disturbance co-exist.

Professor Philip J Cowen, MD University of Oxford Oxford, $U K$

1 Schoenthaler SJ, Bier ID. The effect of vitamin-mineral supplementation on juvenile delinquency among American schoolchildren: a randomized, double-blind placebocontrolled trial. J Altern Complement Med 2000;6:7-17.

2 Rutter M, Giller H, Hagell A. Antisocial behaviour by young people: a major new review. Cambridge: Cambridge University Press, 1998. 